\title{
Uso do Rorschach na Investigação do Abuso Sexual Infantil ${ }^{1}$
}

\author{
Silvana Alba Scortegagna ${ }^{2}$ \\ Universidade de Passo Fundo, Passo Fundo-RS, Brasil \\ Anna Elisa de Villemor-Amaral \\ Universidade São Francisco, Itatiba-SP, Brasil
}

\begin{abstract}
Resumo: A escolha de métodos apropriados para identificar crianças vítimas de abuso sexual é uma prática necessária no cenário forense. Entre os instrumentos de avaliação psicológica, o Método de Rorschach demonstra especial utilidade para esta finalidade. Visando a ampliar e fundamentar essas ideias, este estudo teve como objetivo verificar a extensão e importância da utilização do Rorschach como instrumento de avaliação psicológica de vítimas de abuso sexual. Para tanto, procedeu-se à busca bibliográfica eletrônica de artigos indexados. Os artigos revisados derivam de estudos desenvolvidos no Brasil, Cuba, Argentina, Chile, Estados Unidos da América e Canadá. Os principais achados atestam a validade do Rorschach em casos de suspeitas de abuso sexual e na investigação das consequências do abuso, e suas possíveis contribuições na área, o que confere ao instrumento propriedades necessárias para responder de forma segura nesse contexto.
\end{abstract}

Palavras-chave: avaliação psicológica, teste de Rorschach, abuso sexual, saúde pública

\section{The Use of the Rorschach Method in the Investigation of Sexual Abuse of Children}

\begin{abstract}
The choice of adequate methods to identify children who were victims of sexual abuse is a necessary practice in the forensic scenery. Among the psychological assessment instruments, the Rorschach test shows special usefulness for this purpose. Aiming at the broadening and foundation of these ideas and seeking to verify the extension and importance of the use of this instrument in psychological evaluation of the victims of sexual abuse. To do so, we preceded to an electronic bibliographic research of indexed papers of studies developed in Brazil, Cuba, Argentina, Chile, United States of America and Canada. The main findings certify the validity of Rorschach in cases where sexual abuse is suspected and in the investigation of its sequels, and its possible contributions to the area, which confers to the instrument the necessary properties to answer in a safe way within this context.
\end{abstract}

Keywords: psychological assessment, Rorschach test, sexual abuse, public health

\section{Uso del Rorschach en la Investigación del Maltrato a los Niños}

Resumen: La elección de métodos apropiados para identificar niños víctimas de abuso sexual es una práctica necesaria en el escenario forense. Entre los instrumentos de evaluación psicológica, el método de Rorschach ha demostrado su utilidad especial para esa finalidad. Con el objeto de ampliar y fundamentar esas ideas, se busca verificar la extensión y la importancia de la utilización de este instrumento en la evaluación psicológica de víctimas de abuso sexual. Para esto, se procedió a la búsqueda bibliográfica electrónica de artículos indexados de estudios desarrollados en Brasil Cuba, Argentina, Chile, Estados Unidos de América y Canadá. Los principales resultados certifican la validez de Rorschach en casos de sospechas de abuso sexual y en la investigación de las consecuencias del abuso y sus posibles contribuciones en esta área, lo que confiere al instrumento propiedades necesarias para contestar de forma segura en este ámbito.

Palabras clave: evaluación psicológica, test de Rorschach, abuso sexual, salud pública

A prática da avaliação psicológica para o diagnóstico e o reconhecimento de indicadores emocionais em crianças vítimas de abuso sexual é frequente no âmbito forense. Nesse contexto, a avaliação deve fornecer informações cientificamente fundamentadas de modo a orientar a tomada de decisão em processos judiciais de várias naturezas. Em casos de disputa de guarda em Vara de Família, pode auxiliar a definir

\footnotetext{
${ }^{1}$ Artigo derivado da Tese de Doutorado da primeira autora sob a orientação da segunda, defendida no Programa de Pós-graduação em Psicologia da Universidade São Francisco.

${ }^{2}$ Endereço para correspondência:

Silvana Alba Scortegagna. Universidade de Passo Fundo. Curso de Psicologia/IFCH. Campus I, BR 285, Bairro São José. Caixa Postal 611. CEP 99.001-970. Passo Fundo-RS, Brasil.E-mail: silvanalba@upf.br
}

o guardião legal, como também a busca da veracidade ou não de determinadas acusações para orientar ações de intervenção junto às vítimas e suas famílias.

Com esse propósito, a observação e o uso de entrevistas e de diversos testes são fundamentais na compreensão das características de personalidade e da dinâmica emocional que invade o abuso sexual e os principais envolvidos. Crianças que sofrem abuso sexual são comumente descritas como reservadas e defensivas, com menor disponibilidade para falar sobre suas dores internas e sobrevivem em ambientes familiares disfuncionais e negligentes (Blatt, 1975).

Um importante fator a ser considerado é que os papéis parentais, ao circular na tríade pai-mãe-criança, revelam que nem a mãe e/ou o pai conseguem ocupar suas funções, deixando uma lacuna no processo de interdição. Nesse sentido, 
instauram-se dificuldades na diferenciação de seus membros, e é justamente essa falta de discriminação, resultante da impossibilidade de instauração da função-pai, que deveria constituir o limite e determinar os parâmetros entre o eu e o outro, que promove a relação incestuosa (Bollas, 1992).

Outro aspecto relevante é a participação de mães na relação incestuosa pai/filha, que pode ir desde o favorecimento da ocorrência de estímulos sexuais mais sutis até o contato sexual efetivo, configurando-se em um conluio perverso com o abusador. Essa forma de funcionamento é evidenciada no Rorschach no Sistema Compreensivo (Wald, Archer, \& Winstead, 1990) em elevados escores de respostas de forma distorcida (X-\%) em um Perceptual-Thinking Index (PTI) positivo, sugerindo grave transtorno do pensamento com déficits no teste de realidade; elevações no Índice de Depressão (DEPI), de respostas de sombreado dimensão $(\mathrm{V})$, de sombreado difuso $(\mathrm{Y})$, revelando propensão à autoinspecção ruminativa e à ambivalência emocional; ainda, os indicadores de sombreado textura $(\mathrm{T})$ e a proporção do tipo de vivência (EB) denotam distanciamento nas relações interpessoais e ineficiência de recursos para a resolução de problemas (Exner, 2003). Com isso, é possível considerar que mães de vítimas de abuso sexual processam informações de forma ineficiente, são negligentes e falham em realizar a função materna necessária.

Nesse cenário, são comuns as mães que delegam suas atribuições às próprias filhas, levando-as a assumir suas funções. Entretanto, na medida em que isso ocorre, as mães são invadidas por ressentimentos de hostilidade e de ódio e pelo desejo de que suas filhas sejam punidas e humilhadas por se apoderarem indevidamente de seu lugar (Forward \& Buck, 1989). Nessas situações, observa-se claramente a dinâmica ambivalência (amor-ódio) entre mãe-filha nos casos de abuso sexual.

Essa ambivalência é dinamicamente vivida por meio dos mecanismos de projeção e introjeção. A introjeção possibilita à criança criar uma representação do mundo externo em seu aparelho psíquico, organizar o caos pulsional e dialetizar os desejos do outro - a mãe. Em situações de abuso sexual, a criança introjeta o desejo incestuoso da mãe e, assim, pode aceitar o lugar imposto por ela, como também utilizar essa situação como vingança, apropriando-se daquilo que deveria ser objeto de desejo sexual da mãe - o pai. Percebe-se, então, a importância de se considerar a rivalidade existente entre mãe e filha. Por outro lado, a projeção é um recurso que permite lidar com o ódio resultante da frustração e do desprazer. Nos casos de abuso sexual, quando isso não ocorre, o ódio pode permanecer no psiquismo e mobilizar a emergência de condutas autodestrutivas da criança contra si mesma.

Não raro, pode ainda ocorrer que, em sua história de origem, as mães de vítimas de abuso sexual infantil tenham igualmente sofrido abuso sexual. Dessa maneira, passam a utilizar a negação do incesto de suas filhas, pois a repetição do ato incestuoso entretece um alívio inconsciente, na medida em que as mantém na posição de filhas amadas pelo pai que ora aparece projetado na figura do marido. Diante do exposto, pode-se melhor entender a dificuldade da mãe em dar credibilidade e apoio à filha, sua posição em permanecer ao lado do parceiro e o uso que faz da negação para defender-se contra o reviver de sua própria situação dolorosa (Bollas, 1992).

Desse modo, por mais esforços que a vítima venha empreender para revelar o abuso, em famílias incestuosas a criança é intimada a manter o ato abusivo como um segredo de família, constituindo-se na Síndrome Conectora de Segredo e Adição (Furniss, 1993). Assim, se, por um lado, a manutenção do silêncio beneficia a perpetuação das relações familiares incestuosas e incrementa a sua adição, por outro, a possibilidade de se intervir para abrir um espaço para a denúncia pode auxiliar a criança e sua família. Vale ainda dizer que os efeitos do silêncio favorecem o isolamento da vítima, além de aumentar a introjeção da culpa que a faz se sentir responsável pelo infortúnio, agravando-se, assim, o impacto traumático e suas consequências.

Desde Freud (1920/1976) se tem conhecimento de que é traumático tudo o que gera uma grande quantidade de excitação que impede a circulação psíquica. Assim, o abuso sofrido pela criança é um fato objetivo que se torna traumático e impossível de ser processado psiquicamente, resultando para a criança em uma realidade intolerável, que gera excesso de excitação, a qual pode encontrar uma saída na descarga motora e em manifestações recorrentes de angústia. Tais manifestações buscam por via da descarga metabolizar esse excesso e encontrar formas de simbolizar essa vivência.

A obstaculização do pensamento decorre justamente da incapacidade da criança para suportar a percepção da sua dura realidade e o sofrimento dela advindo. Como consequência, a capacidade de pensar, de investigar, de simbolizar, torna-se precária e a compreensão e o ajustamento ao mundo são prejudicados. São frequentes os danos no desenvolvimento do pensamento e na aprendizagem, que não estão relacionados a uma deficiência nas estruturas cognitivas, mas a uma inibição sintomática como tentativa de remover uma situação de perigo geradora de ansiedade para o ego, que é conhecer a própria história.

É assim que a emergência de sintomas de ansiedade, distúrbios do sono, transtorno de stress pós-traumático e comportamento sexual inapropriado se tornam habituais. Entretanto, a presença ou não de determinados sintomas depende do período evolutivo, de maneira que alguns efeitos podem se apresentar durante a infância e outros, em outra etapa (Finkelhor, 1995). Por exemplo, em crianças em idade escolar os sintomas predominantes incluem comportamento regressivo e agressivo, hiperatividade, distúrbios do sono e problemas escolares. $\mathrm{Na}$ adolescência, os sintomas típicos são depressão, isolamento, abuso de substâncias, queixas somáticas, comportamento sexual inadequado, autoagressão e tentativas de suicídio.

Torna-se importante ressaltar, portanto, que a sintomatologia apresentada pode decorrer da força traumática que 
procede da incapacidade de dar figurabilidade à dor psíquica, isto é, de dar uma atribuição de sentido ou obter uma captura no mundo representacional do sujeito. Diante da ação do traumático, da ausência de palavras, provocada pela força do irrepresentável, o indivíduo pode tentar dar fim à própria vida. As vivências de maus-tratos podem gerar repetições, como tentativas de criar uma nova representação do irrepresentável, mas que, diante dessa impossibilidade, cedem espaço ao ato, como única alternativa de descarga. Os atos dirigidos contra si mesmo podem indicar a inter-relação existente entre vivências de excesso, consequentemente, entre trauma, dor psíquica e ato (Macedo \& Werlang, 2007).

Outra forma de a criança manifestar o seu sofrimento psíquico é a tentativa que por vezes pode fazer de transformação da realidade, mediante a recusa ou desmentida. Como uma forma de defesa, o termo desmentida consiste na recusa do sujeito em reconhecer a realidade de uma percepção intolerável e traumatizante (Laplanche \& Pontalis, 1992). Depreende-se, portanto, que, quanto mais insuportável a realidade objetiva, mais as crianças vítimas de abuso sexual tendem a se afastar dela na tentativa de desmentir o evento traumático. Isso dificulta não apenas a identificação das vivências de abuso, mas também o auxílio da intervenção para a elaboração necessária.

Não é difícil compreender que a criança se utilize defensivamente da recusa da realidade quando se percebe que o que funda o traumático ou a dor psíquica, muito além das eventuais dores ou marcas físicas decorrentes do abuso, é a dor de perceber que seus primeiros objetos de amor agem em prol de seu aniquilamento. Nessa perspectiva, a compreensão da dinâmica abusiva poderia justificar a não utilização de medidas de autorrelato e o direcionamento para escolhas de técnicas projetivas com vistas a tocar aspectos do funcionamento que não são facilmente acessíveis, em razão do trauma vivido e que os sujeitos estão impossibilitados ou não são capazes de relatar (Blatt, 1975).

Indo um pouco além, pode-se comparar o que Conte (2008) problematiza ao discutir a prática do Depoimento Sem Dano sob a escuta psicanalítica versus a escuta do direito e a utilização dos testes projetivos versus os de autorrelato na avaliação psicológica na vitimização sexual. O Depoimento Sem Dano é uma prática que visa reduzir o dano das inúmeras oitivas às quais as crianças são submetidas em processos de abuso sexual e para auxiliar na produção de provas. Nesta técnica crianças e adolescentes são ouvidos por juízes, promotores, advogados e serventuários da Justiça, que podem interagir durante o testemunho. Psicólogos e assistentes sociais desenvolvem basicamente a função de intermediação, isto é, repassam as perguntas à vítima com vistas a obter "a verdade" necessária. Para o registro material das provas a sala para esta finalidade integra equipamentos de áudio e vídeo e, após a transcrição desses documentos, uma cópia da gravação é anexada ao processo.

Retomando as considerações da referida autora, a demanda de validade da fala da criança, quando exposta a um testemunho, evidencia um paradoxo, pois esta precisa revelar e esconder. Revelar o solicitado quanto ao inquérito significa desvendar a verdade objetiva, e esconder o acontecido constitui-se em ocultar a vivência subjetiva de dor, vergonha, embaraço e passividade. Assim, nem tudo pode estar disponível no nível simbólico da palavra, e quando não se está respeitado o tempo do que não pode ser revelado por não haver possibilidade de elaboração psíquica, o que pode ocorrer é a revitimização.

Ao abordar o aspecto imperativo do inquérito de uma situação traumática, Conte ainda declara que, além de provocar a atualização da intensidade da excitação experimentada frente ao abuso, revitimiza a criança que luta para suportar psiquicamente essa vivência. Se ainda o inquérito for realizado muito tempo após o abuso, pode igualmente trazer problemas à criança, pois o traumático inscrito e não simbolizado podem tomar vias de formação de sintomas no corpo, da descarga em atos ou da clivagem do ego, como foi anteriormente observado.

Portanto, a intervenção terapêutica nesses casos encontra-se bem fundamentada, pois convocar a criança a discorrer sobre o abuso sofrido, sem dar destino a este traumático, é igualmente violência. Se, do ponto de vista da vítima, pode-se chegar a tais constatações, ficam ainda as perguntas: Qual seria a função de um psicólogo, a de escuta ou a de inquirição? Como proceder à avaliação psicológica nesse contexto?

Diferentemente da proposta de Depoimento Sem Dano, a prática do psicólogo na escuta psicanalítica do sofrimento da criança vitimizada consiste, sobretudo, na possibilidade de oferecer meios para que uma inscrição traumática como o abuso possa adquirir um significante, uma representação, uma fala, para que esta criança então possa transformar o excesso vivido no real do corpo, em uma expressão de símbolo, dando início à elaboração psíquica. Esse processo não se efetiva somente por meio da construção de uma verdade histórica, mas de um histórico vivencial.

Diante destas ponderações, pode-se supor que o uso de instrumentos de autorrelato ocupa um lugar similar ao inquérito proposto no Depoimento Sem Dano, quando confronta de forma mais direta e objetiva a criança com a sua dor e sofrimento. Nessas circunstâncias, a importância da utilização de recursos como o Método de Rorschach parece indispensável na medida em que pode dar início à elaboração simbólica por meio de formas de expressão indiretas, as quais a criança tem liberdade de eleger. Ao compor imagens ou construir formas a partir de estímulos pouco estruturados, a criança se depara com as vivências inscritas a partir de sua experiência, o que permite compreender tanto a sua dinâmica afetiva como as suas possibilidades cognitivas (Güntert, 2000).

De sobremaneira, o Rorschach desempenha um papel valioso na descrição da interação complexa e dinâmica entre os domínios psicológico, biológico e social, comumente solicitada, no âmbito legal, em situações de vitimização. Porém, embora o instrumento atenda aos rigores do escrutínio forense de forma consistente, Gacono, Evans e Viglione (2008) 
destacam a importância de o examinador selecionar as variáveis do Rorschach que são diretamente relevantes às questões necessárias em cada situação, as quais ao mesmo tempo apresentem normas e construtos de validade de pesquisa para a população forense específica.

Com isso, os autores destacam a necessidade de o examinador não apenas estar familiarizado com a validade da pesquisa relacionada às variáveis do Rorschach no Sistema Compreensivo, mas também conhecer os dados forenses comparativos relevantes e as implicações para essa prática. Dentre vários estudos disponíveis destacam-se os de Bannatyne, Gacono e Greene (1999) e de Gacono e Meloy (1994), para pacientes psiquiátricos forenses e pacientes antissociais e psicopatas, e os de Gacono, Meloy e Bridges (2000) para ofendedores sexuais.

Além disso, o Método de Rorschach tem especial utilidade em situações nas quais o respondente pode não ter vontade ou ser incapaz de se engajar ao exame. Entre elas se incluem as avaliações compulsórias para responder às questões do âmbito forense criminal e de custódia que envolvem componentes de relacionamentos adversos. Nessas condições, a manipulação da resposta a partir do autorrelato pode comprometer ou até invalidar os achados confiáveis (Bannatyne, Gacono, \& Greene, 1999).

Convém lembrar que as avaliações por meio de métodos como o Rorschach, também chamados de autoexpressão, visam buscar mais do que se pode evidenciar pelo autorrelato, ou por sintomas, pois para emitir diagnósticos nosográficos as entrevistas estruturadas, as escalas e os inventários são eficientes (Santoantonio \& Antunez, 2010; Villemor-Amaral, 2008). Nas avaliações compulsórias, entretanto, os comportamentos manifestos se caracterizam muitas vezes por simulações, o que demanda o uso de instrumentos hábeis em fornecer informações além daquelas que os controles volitivos de um indivíduo permitem (Villemor-Amaral, 2009). Tais benefícios são obtidos com o Rorschach, que, pela sua natureza, possibilita ao sujeito a livre expressão de características de sua personalidade sem que se sinta ameaçado pela objetividade de questionários ou inventários.

Contudo, diferentemente das medidas de autorrelato, o Rorschach requer um trabalho extensivo e uma prática supervisionada para tornar-se eficiente na administração e na aferição dos escores básicos. Em decorrência dos altos padrões de complexidade requeridos na avaliação forense, os especialistas no Rorschach devem ter extensa experiência em codificar protocolos para uma prática mais segura. Além disso, uma boa aplicação e codificação não garantem a maestria no Rorschach, que inclui interpretações habilidosas.

Toma-se como exemplo o estudo de Weiner, Exner e Sciara (1996) para demonstrar a complexidade do que se tem exposto. $\mathrm{Na}$ análise de 7.934 processos julgados em tribunais com o auxílio dos resultados do Rorschach, os pesquisadores constataram que apenas sete haviam sido contestados. De forma invariável, onde as contestações foram sustentadas, não foram as propriedades psicométricas do Rorschach desacreditadas, mas as interpretações do psicólogo (Viglione $\&$ Meyer, 2008). Entre os principais problemas encontrados observou-se que, diante da corte, as interpretações eram amplas demais, usadas para demonstrar um crime que foi cometido; específicas demais, apenas para auxiliar na formulação do diagnóstico sem a ligação com o tópico forense; ou completamente irrelevantes ao problema legal. Dessa forma, as três situações implicam falhas na formação do psicólogo em detrimento das qualidades do instrumento.

Apesar de todos esses indicadores que acenam para a legitimidade do Método de Rorschach no contexto forense e em situações de vitimização sexual, quando é investigado o uso desse instrumento para este fim, observa-se uma marcante escassez de estudos brasileiros quando comparados à quantidade de estudos desenvolvidos em outros países, embora também aí não sejam muito numerosos. Uma hipótese para isso pode se apoiar em, pelo menos, duas condições fundamentais, uma relacionada a complexidade das questões que permeiam a vitimização sexual e a avaliação compulsória e outra, à natureza do instrumento.

Visando ampliar e fundamentar essas ideias, este estudo teve como objetivo verificar a extensão e importância da utilização do Rorschach como instrumento de avaliação psicológica de vítimas de abuso sexual. Desse modo, foi realizada a busca bibliográfica eletrônica de artigos indexados em bases de dados LILACS, Index-Psi Periódicos, Banco de Teses da CAPES, PePSIC, a partir das palavras-chave Rorschach e abuso sexual infantil, os quais foram desenvolvidos no Brasil, Cuba, Argentina, Chile, Estados Unidos da América e Canadá, entre 1988 e 2008.

Os trabalhos encontrados são escassos, principalmente nos últimos anos. De forma específica, podem ser agrupados em aqueles que procuram usar o Rorschach para tentar determinar ou confirmar as vivências traumáticas ou as suspeita de abuso e os que visam identificar suas prováveis consequências para o desenvolvimento psíquico de crianças com vistas a planejar medidas de intervenção. Trata-se de objetivos diferentes, mas que convergem no sentido de oferecer suporte a decisões judiciais quanto ao desenvolvimento de intervenções terapêuticas às pessoas envolvidas. Daí a necessidade de se examinarem as principais conclusões dessas duas perspectivas.

\section{Uso do Rorschach na Avaliação do Trauma e de Suspeita de Abuso Sexual}

Os primeiros estudos com o uso do Rorschach em vítimas de trauma foram desenvolvidos com indivíduos em tempos de guerra. Shalit (1965) administrou o Rorschach em vinte homens em serviço militar na marinha enquanto enfrentavam uma severa tempestade no mar. Esse estudo foi o primeiro a demonstrar o aumento no movimento inanimado $(m)$, sendo, posteriormente, consistentemente comprovado em pesquisas de trauma. Em 1984 com base no recém-criado diagnóstico de Transtorno de Estresse Pós-Traumático (TEAP), Salley e Teiling (1984) estudaram veteranos combatentes no Vietnã e 
tornaram-se os primeiros pesquisadores a documentar intrusões traumáticas com base no Rorschach.

Cerney (1990) relatou dois modos distintos de respostas no Rorschach: as que revelam características de pacientes "inundados pelo trauma" (flooded) e as de pacientes "evitativos" (avoidant). Assim, em razão da possibilidade de resposta bifásica ao trauma, os protocolos do Rorschach são propensos a revelar tanto sinais de inundação quanto de reserva. As verbalizações reveladoras de sentimentos de constrição apresentam-se com nenhum determinante de cor e as de inundação, com nenhuma modulação de cor.

Estudos posteriores foram realizados com o objetivo de verificar um padrão bifásico de resposta ao trauma (Brand, Armstrong, \& Loewenstein, 2006; Kaser-Boyd, 1993). Os achados incluíram labilidade emocional $(\mathrm{CF}+\mathrm{C}>\mathrm{FC})$ adjacente com baixa proporção afetiva $(<.05)$. Os sentimentos de evitação do trauma foram observados na combinação de um Lambda (L) alto, baixo número de respostas $(\mathrm{R})$ e baixos valores do quociente afetivo (Afr) associados a um significativo Índice de Conteúdo Traumático (TC), cor pura (C) e cor com forma secundária (CF), e Perceptual-Thinking In$\operatorname{dex}(\mathrm{PTI})$. Constatou-se ainda, a presença de protocolos tanto com sinais de "constrição" quanto de "inundação" como também a existência de protocolos com somente um desses sinais, dependendo da fase em que se encontrava o indivíduo no período da avaliação.

No sentido de ampliar e aprofundar mais essa compreensão destacam-se os trabalhos que evidenciam os sinais de evitação do trauma e os que revelam a presença de sentimentos de inundação. Os sinais de evitação são descritos em dois padrões de respostas de veteranos de guerra ao Rorschach marcados por uma falta de respostas afetivas e um baixo índice de resposta. Os baixos valores do quociente afetivo (Afr) e a baixa porcentagem de determinantes mistos (Blends) remetem a uma paralisia emocional (Kaser-Boyd, 1993), ao passo que o baixo número de respostas (R) e um Lambda (L) alto são compreendidos como manifestações de evitação cognitiva e, quando combinadas, podem produzir um tipo de vivência (EB) baixo, comumente encontrado em contextos de vitimização (Levin \& Reis, 1996).

Em populações com desordens dissociativas constata-se um número incomum de sujeitos superintrovertidos. Por outro lado, em grupos de trauma não dissociativos se observa a presença de um padrão extratensivo (Armstrong \& Loewenstein, 1990). Depreende-se, portanto, que a dissociação pode estar relacionada com a presença de alguns determinantes únicos que indicam um sinal de distanciamento cognitivo e emocional, como, por exemplo, resposta Forma Dimensão (FD) (Armstrong, 1991). Esses achados são consistentes com a teoria do desenvolvimento quando evidencia que a dissociação permite à criança se distanciar da sobrecarga de emoção e evadir-se para o mundo imaginário, mais gratificante do que o mundo real (Armstrong, 1994).

Mais recentemente, Brand et al. (2006) investigaram 100 pacientes internados com desordens psiquiátricas e severa dissociação utilizando-se do Rorschach e evidenciaram importantes sinais de evitação traumática. Em mais de $40 \%$ da amostra foi obtido $\mathrm{R}<14$, um dado significativo quando comparado aos grupos de controle relevantes. Os autores compreenderam esse padrão de comportamento como uma tentativa de "limitar e escapar das associações dolorosas". Sinais adicionais de hesitação foram encontrados na alta prevalência de respostas Forma Dimensão (FD); na maior frequência de indivíduos introvertidos, o que sugere a presença de processos de intelectualização e obsessão; nos valores baixos do quociente afetivo (Afr), o que representa uma paralisia emocional, e nos índices elevados de respostas de movimento humano $(M)$, o que indica o uso da fantasia como meio de se resguardar.

Indivíduos em que a experiência traumática foi recente ou especialmente assustadora comumente apresentam sinais de inundação do trauma. Em protocolos do Rorschach tendem a atribuir detalhes desnecessários, como se fossem incapazes de defender contra imagens de perigo. A inundação traumática pode ser observada nas respostas de cor relativamente não estruturadas ( $\mathrm{CF}+\mathrm{C}>\mathrm{FC}$ ) e $\mathrm{EB}$ extratensivo; no afeto doloroso expresso na predominância de respostas de sombreado, particularmente sombreado difuso (Y) e sombreado dimensão (V) (Salley \& Teiling, 1984); em altos escores da Nota D negativa e Nota D ajustada (AdjD), em razão dos efeitos danosos da inundação; na presença significativa do movimento inanimado (m) e um Índice de Hipervigilância (HVI) positivo, que refletem sentimentos de desespero em face das forças de sobrecarga e hipersensibilidade ao perigo maior (Levin, Lazrove, \& van der Kolk, 1999).

É interessante destacar que a dissociação, geralmente presente em períodos de evitação, pode igualmente aparecer na fase de inundação, sob a forma de flashbacks. Estudos têm procurado identificar os flashbacks por meio da análise do conteúdo. Desde as primeiras pesquisas com o Rorschach em populações vítimas de guerra, os pesquisadores observaram a presença de conteúdo traumático (Leifer, Shapiro, Martone, \& Kassem, 1991). Armstrong (1991), por exemplo, desenvolveu com amostras de pacientes com desordens dissociativas um Índice de Conteúdo Traumático $(T C)$, que consiste na soma das respostas de conteúdo sexo $(\mathrm{Sx})$, sangue $(\mathrm{Bl})$, anatomia $(\mathrm{An})$, mórbido (MOR), movimento agressivo (AG) dividido pelo número total de respostas (TC/R). Um TC/R de.3 ou mais foi hipotetizado como sugerindo intrusões traumáticas. Kamphuis, Kugeares e Finn (2000) documentaram a habilidade do TC/R em distinguir entre pacientes com abuso sexual confirmado daqueles livres desse infortúnio.

Com essa preocupação, pesquisadores buscaram diferenciar as respostas no Rorschach de crianças e adolescentes vítimas de abuso sexual em comparação com as não-vítimas. Einbender e Friedrich (1989) avaliaram dados do Rorschach relativos ao funcionamento cognitivo, emocional, social e preocupação sexual. Eles constataram diferenças entre os grupos no funcionamento cognitivo, social 
e na preocupação sexual; ainda, os relatos das vítimas resultaram em um elevado índice de conteúdo mórbido, sexual e de respostas incomuns.

Outros estudos levam a conclusões semelhantes ao demonstrar que as vítimas exibem mais respostas de conteúdo não usual e incomum, como sexo e sangue. Kendall-Tackett, Williams e Finkelhor (1993) reportam a alta frequência desses conteúdos como sendo o mais robusto indicador do abuso sexual, o que revela aspectos de angústia e demonstra indicativos de a criança de sofrer sérios danos, de ser invadida e machucada.

Ao estudar o funcionamento psicológico dessas crianças, Leifer et al. (1991) compararam as respostas de meninas com e sem história de abuso. Descobriram que as vítimas diferiam nos elevados escores de respostas de distorção perceptiva (X-\%); no uso predominante de características de sombreado; no Índice de Depressão (DEPI) positivo; na elevada soma dos códigos especiais, relativos a características estranhas na verbalização, e de conteúdos incomuns, hostis; na diminuição no número de respostas $(\mathrm{R})$; e uma diferença quanto à frequência de respostas com conteúdos de objetos penetrando outros. Resumindo, as vítimas demonstraram pensamento mais perturbado, um nível mais elevado de estresse relativo às suas habilidades adaptativas; descreveram os relacionamentos humanos de forma mais negativa e demonstraram mais preocupação com a sexualidade do que o grupo comparativo.

Conforme o exposto é possível supor que em suspeita de abuso esses indicadores podem contribuir para confirmação do fato, realçando a importância do Rorschach nesse contexto. Pretende-se a seguir verificar a validade do Rorschach para mapear as consequências do abuso e atender as suas implicações.

\section{Uso do Rorschach na Avaliação das Consequências do Abuso Sexual}

Encontram-se na literatura estudos com adultos que foram crianças vítimas de abuso sexual, os quais demonstram as consequências indeléveis de tais experiências, reforçando a necessidade de intervenções apropriadas. Cerney (1990) e Friedrich, Jaworski, Huxsahl e Bengtson (1997) reportaram, de um modo geral, um forte componente dissociativo no Rorschach verificado nas respostas de conteúdo mórbido e sexual e no aumento do índice de respostas incomuns. Investigações posteriores apoiam esses achados ao constatar a presença de respostas dissociativas no Rorschach de vítimas de abuso sexual, o que indica maior vulnerabilidade destes indivíduos a apresentar distúrbios envolvendo o soma e o self.

Chagnon (2008) chamou a atenção para as características de protocolos dissociativos e com indicadores de um prejuízo primário na construção da identidade, onde insurgem relatos de conteúdos mal-diferenciados e pouco integrados. De maneira recorrente, são comuns as dificuldades em diferenciar formas precisas, que aparecem frequentemente interpenetradas; além de ocorrer verbalizações quanto ao interior do corpo, utilizando-se como recurso as imagens anatômicas e uterinas, o que remete a uma fragilidade das fronteiras dentro-fora ou interno-externo. É interessante observar que as imagens evocadas podem estar testemunhando o dano corporal e psíquico sofrido pelo sujeito, o que corresponde a um sentido de não ser, como um self desmantelado (Meltzer, 1984), ou ainda, de não ser diferenciado. Tais respostas indicam também um profundo sentimento de fragilidade e desproteção.

Kendall-Tackett et al. (1993) e Leifer e al. (1991) buscaram identificar características de abuso sexual infantil associada ao desenvolvimento de danos mais sérios. Os resultados foram obtidos pelas análises dos relatórios psiquiátricos e de seis variáveis do Rorschach: as respostas de movimento humano de qualidade pobre; os escores elevados de códigos especiais; as respostas mórbidas; as respostas acromáticas; as respostas de sombreado difuso e o percentual de respostas de forma precisa rebaixado. Os autores concluíram que o abuso na primeira infância estava relacionado às mesmas variáveis do Rorschach, caracterizando cognição perturbada e senso de si mesmo danificado. Os distúrbios psicológicos mais graves foram relacionados à presença de mais de um perpetrador, à pouca idade da vítima e aos frequentes episódios de abuso.

Nesse contexto, observa-se o emprego do Rorschach na Argentina na busca de elementos para o diagnóstico e a determinação de dano mental. Gravenhorst (2002), ao analisar protocolos de vítimas de abuso sexual, constatou a presença de $\mathrm{F} \%$ e $\mathrm{F} \%$ estendida no âmbito esperado, indicando esforços do ego em ser objetivo e lógico. O estudo corroborou com os achados mencionados no que diz respeito ao $\mathrm{F}+\%, \mathrm{~F}+\%$ estendida e Índice de Realidade (IR) diminuídos, o que sugere patologia severa associada ao funcionamento do ego não operativo com falha na adaptação à realidade, respostas de forma, indicadores de rigidez defensiva, dissociação e superadaptação.

Outros elementos significantes encontrados pela autora foram respostas de danos associados com a sexualidade e de conteúdo de esqueleto; presença de conteúdo de sangue; respostas sexuais diretas e de figuras masculinas perseguidoras, agressivas e violentas. Entre os fenômenos especiais foi verificado: (a) ação de tolerância no presente ou no passado como um indicativo de ter suportado passivamente uma ação violenta; (b) presença de respostas de conteúdo mórbido (MOR) na identificação de objetos danificados, destruídos, quebrados, mortos; (c) respostas de complexo oral sádico associado com sexualidade; (d) anulação da Consciência de Interpretação com Auto-referências negativas na mesma resposta, indicadores de dano psicológico severo, perda de limites e julgamento da realidade.

Como se pode observar, os poucos estudos internacionais e a inexistência de estudos brasileiros com o Rorschach na investigação do abuso sexual infantil indicam a 
necessidade de realização de estudos de validação e de diagnósticos com esse instrumento em nosso meio. Com esse propósito, Scortegagna e Villemor-Amaral (2009) avaliaram 76 crianças e adolescentes, de ambos os sexos, com idades entre 10 e 14 anos, divididos em dois grupos distintos: um grupo de vítimas de abuso incestuoso e outro de não-vítimas. As autoras utilizaram uma ficha sociodemográfica e o Rorschach no Sistema Compreensivo. Foram encontradas, além de seqüelas no desenvolvimento cognitivo, diferenças significativas entre os indicadores do Rorschach nas respostas de movimento inanimado $(\mathrm{m})$ e de conteúdo sangue $(\mathrm{Bl})$. As respostas de conteúdo anatômico (An), mórbido (MOR), sexual (Sx) e de qualidade formal imprecisa e não convencional (FQ-) demonstraram pontuações mais altas no grupo de vítimas. Constatou-se que a presença de uma autopercepção distorcida e de uma autoestima rebaixada nas vítimas é decorrente do processo de vitimização. Esses resultados foram considerados significativos, uma vez que corroboram com os dados obtidos em estudos estrangeiros e demonstram a validade do uso do Rorschach nesse contexto.

\section{Considerações Finais}

Refletir sobre o uso do Rorschach na investigação do abuso sexual infantil conduz a muitas inquietações. Em princípio, vale lembrar que a maior parte da literatura enfatiza a ocorrência do incesto em um funcionamento familiar perturbado, o que leva a pensar que, seja pelas desordens familiares, seja pela fragilidade da criança, as inúmeras dificuldades de se romper com a dinâmica familiar incestuosa na revelação do abuso sexual infantil são delicadas e complexas.

Diante dessa complexidade e da necessidade de melhor contribuir na elucidação das denúncias ou nas implicações das ocorrências, buscam-se recursos nas técnicas psicológicas apropriadas e nos métodos de investigação válidos. Constata-se, então, que a escuta psicanalítica, por se amparar em recursos que melhor demonstram a compreensão da vivência traumática e dos fatores protetores significantes, pode ser de grande auxílio. Esses elementos, ainda, são úteis para o desenvolvimento dos processos de avaliação psicológica e para os de intervenção que objetivam a integração do evento traumático.

Entre os métodos de investigação psicológica, o Rorschach destaca-se como um dos instrumentos de melhor eficiência para esse contexto. Além de suas propriedades psicométricas que evidenciam a consistência de sua validade e confiabilidade, quando bem administrado, é uma ferramenta valiosa que incrementa a prática forense. Aliado aos seus escores e proporções, oferece a oportunidade de observar o comportamento em resposta a um estímulo novo e complexo. É interessante observar que, ao interpretar formas fortuitas, as imagens evocadas pelas crianças vitimizadas podem estar testemunhando seu dano corporal e psíquico, como já observado. Com isso o Rorschach acrescenta ainda uma dimensão adicional às entrevistas face a face, componentes essenciais das avaliações forenses.
Há, portanto, grande convergência nos resultados de pesquisas com o Rorschach tanto em crianças quanto em adultos. Embora ocorram variações nas perspectivas metodológicas entre os diversos autores citados, todos admitem que conteúdos de sexo, sangue, com características mórbidas e indicadores de falhas na percepção da realidade, são elementos que surgem em protocolos de sujeitos com vivências de abuso sexual recente ou mais remoto. Isso permite ao Rorschach o status de instrumento que ajuda a identificar situações de abuso para além do que pode ser relatado e contribui, também, para estabelecer diretrizes de intervenção em curto e logo prazo no atendimento às vítimas e suas famílias.

Por fim, a pesquisa sólida dos últimos vinte anos inclui uma análise da aceitação do Rorschach nos tribunais e sugere que o instrumento continuará a ser amplamente usado no trabalho clínico e forense. Desse modo, claro está que a pesquisa continuada é necessária para especificar as aplicações e limitações para muitos postulados interpretativos e para o desenvolvimento de tabelas normativas brasileiras para a população forense alvo.

\section{Referências}

Armstrong, J. G. (1991). The psychological organization of multiple personality disordered patients as revealed in psychological testing. Psychiatric Clinics of North America, 14(3), 533-546.

Armstrong, J. G. (1994). Reflections on multiple personality disorder as a developmentally complex adaptation. The Psychoanalytic Study of the Child, 49, 349-364.

Armstrong, J. G., \& Loewenstein, R. J. (1990). Characteristics of patients with multiple personality and dissociative disorders on psychological testing. Journal of Nervous and Mental Disease, 178(7), 448-454.

Bannatyne, L. A., Gacono, C. B., \& Greene, R. L. (1999). Differential patterns of responding among three groups of chronic psychotic forensic outpatients. Journal of Clinical Psychology, 55(12), 1553-1565. doi: 10.1002/(SICI)1097-4679(199912)55:12<1553::AIDJCLP12>3.0.CO;2-1

Blatt, S. J. (1975). The validity of projective techniques and their research and clinical contributions. Journal of Personality Assessment, 39(4), 327-343. doi: 10.1207/ s15327752jpa3904_1

Bollas, C. (1992). A sombra do objeto: Psicanálise do conhecido não-pensado. Rio de Janeiro, RJ: Imago.

Brand, B. L., Armstrong, J. G., \& Loewenstein, R. J. (2006). Psychological assessment of patients with dissociative identity disorder. Psychiatric Clinics of North America, 29(1), 145-168.

Cerney, M. S. (1990). The Rorschach and traumatic loss: Can the presence of traumatic loss be detected from the Rorschach? Journal of Personality Assessment, 55(3-4), 781-789. 
Conte, B. S. (2008). Depoimento sem dano: A escuta da psicanálise ou a escuta do direito? PSICO, 39(2), 219223. Recuperado de http://revistaseletronicas.pucrs.br/ ojs/index.php/revistapsico/article/viewFile/2262/3043

Chagnon, J. Y. (2008). Traumatismo, violência e agressões sexuais. In S. R. Pasian, E. T. K. C. Okino, S. R. Loreiro, \& F. L. Osório (Orgs.), Avaliação da Personalidade: Técnicas e contextos diversos: $V$ Encontro da Associação Brasileira de Rorschach e Métodos Projetivos (Vol. 4, pp. 510-535). Ribeirão Preto, SP: SBRo.

Einbender, A. J., \& Friedrich, W. N. (1989). Psychological functioning and behavior of sexually abused girls. Journal of Consulting and Clinical Psychology, 57(1), 155-157.

Exner, J. E. (2003). The Rorschach: A comprehensive system (4th ed., Vol. 1). New York, NY: Wiley.

Finkelhor, D. (1995). The victimization of children: A developmental perspective. American Journal of Orthopsychiatry, 65(2), 177-193. doi: 10.1037/h0079618

Forward, S., \& Buck, C. (1989). A traição da inocência: $O$ incesto e sua devastação (S. Flaksman, Trad.). Rio de Janeiro, RJ: Rocco.

Freud, S. (1976). Além do princípio do prazer. In Edição standard brasileira das obras psicológicas completas de Sigmund Freud (J. Salomão, Trad., Vol. 18, pp. 13-61). Rio de Janeiro, RJ: Imago. (Original publicado em 1920)

Friedrich, W. N., Jaworski, T. M., Huxsahl, J. E., \& Bengtson, B.S.(1997). Dissociative and sexual behaviors in children and adolescents with sexual abuse and psychiatric histories. Journal of Interpersonal Violence, 12(2), 155 171. doi:10.1177/088626097012002001

Furniss, T. (1993). Abuso sexual da criança: Uma abordagem multidisciplinar, manejo, terapia e intervenção legal integrados (M. A. V. Veronese, Trad.). Porto Alegre, RS: Artes Médicas.

Gacono, C. B., Evans, F. B., \& Viglione, D. J. (2008). Essential issues in the forensic use of the Rorschach. In C. B. Gacono \& F. B. Evans (Eds.), The handbook of forensic Rorschach assessment (pp. 3-20). New York, NY: Routledge.

Gacono, C. B., \& Meloy, J. R. (1994). The Rorschach assessment of agressive and psychopathic personalities. Hillsdale, NJ: Lawrence Erlbaum.

Gacono, C. B., Meloy, J. R., \& Bridges, M. R. (2000). A Rorschach comparison of psychopaths, sexual homicide perpetrators, and nonviolent pedophiles: Where angels fear to tread. Journal of Clinical Psychology, 56(6), 757-777. doi: 10.1002/(SICI)10974679(200006)56:6<757::AID-JCLP6>3.0.CO;2-I

Gravenhorst, M. C. (2002). Rorschach psychodiagnosis of psychic trauma in sexually abused children. In A. Andronikof-Sanglade (Ed.), Rorschachiana XXV: Yearbook of the International Rorschach Society (pp. 7785). Ashland, OH: Hogrefe \& Huber.
Güntert, A. E. V. A. (2000). Técnicas projetivas: O geral e o singular em avaliação psicológica. In F. F. Sisto, E. T. B. Sbardelini, \& R. Primi (Orgs.), Contextos e questões da avaliação psicológica (pp. 77-84). São Paulo, SP: Casa do Psicólogo.

Kamphuis, J. H., Kugeares, S. L., \& Finn, S. T. (2000). Rorschach correlates of sexual abuse: Trauma content and aggression indexes. Journal of Personality Assessment, 75(2), 212-224. doi:10.1207/S15327752JPA7502_3

Kaser-Boyd, N. (1993). Pos-traumatic stress disorders in children and adults: The legal relevance. Western State University Law Review, 20(2), 319-334.

Kendall-Tackett, K. A., Williams, L. M., \& Finkelhor, D. (1993). Impact of sexual abuse on children: A review and synthesis of recent empirical studies. Psychological Bulletin, 113(1), 164-180.

Laplanche, J., \& Pontalis, J. B. (1992). Vocabulário de psicanálise. São Paulo, SP: Martins Fontes.

Leifer, M., Shapiro, J. P., Martone, M. W., \& Kassem, L. (1991). Rorschach assessment of psychological functioning in sexually abused girls. Journal of Personality Assessment, 56(1), 14-28. doi: 10.1207/s15327752jpa5601_2

Levin, P., Lazrove, S., \& van der Kolk, B. (1999). What psychological testing and neuroimaging tell us about the treatment of posttraumatic stress disorder by eye movement desensitization and reprocessing. Journal of Anxiety Disorders, 13(1-2), 159-172.

Levin, P., \& Reis, B. (1996). Use the Rorschach in assessing trauma. In J. P. Wilson \& T. M. Keane (Eds.), Assessing psychological trauma and PTSD (pp. 529-543). New York, NY: Guilford.

Macedo, M. M. K., \& Werlang, B. S. G. (2007). Trauma, dor e ato: O olhar da psicanálise sobre uma tentativa de suicídio. Ágora, 10(1), 86-106.

Meltzer, D. (1984). Dream-life: A re-examination of the psychoanalytical theory and technique. Perthshire, Scotland: Clunie.

Salley, R. D., \& Teiling, P. A. (1984). Dissociated rate attacks in a Vietnam veteran: A Rorschach study. Journal of Personality Assessment, 48(1), 98-104. doi: 10.1207/ s15327752jpa4801_17

Santoantonio, J., \&Antunez,A.E.A. (2010). Ateliê de desenho e Rorschach: Estudo fenômeno-estrutural. Paidéia (Ribeirão Preto), 20(45), 117-122. Recuperado de http:// www.scielo.br/pdf/paideia/v20n45/a14v20n45.pdf. doi: 10.1590/S0103-863X2010000100014

Scortegagna, S. A., \& Villemor-Amaral, A. E. (2009). Autopercepção no Rorschach de vítimas de abuso sexual infantil. Psico, 40(3), 328-336. Recuperado de http:// revistaseletronicas.pucrs.br/ojs/index.php/revistapsico/ article/viewFile/5736/4800

Shalit, B. (1965). Effects of environmental stimulation on the M, FM and $\mathrm{m}$ responses in the Rorschach. Journal of Projective Techniques and Personality Assessment, 29(2), 228-231. doi: 10.1080/0091651X.1965.10120201 
Viglione, D. J., \& Meyer, G. J. (2008). An overview of Rorschach psychometrics for forensic practice. In C. B. Gacono \& F. B. Evans (Eds.), The handbook of forensic Rorschach assessment (pp. 21-53). New York, NY: Routledge.

Villemor-Amaral, A. E. (2008). A validade teórica em avaliação psicológica. Psicologia Ciência e Profissão, 28(1), 98-109. doi: 10.1590/S1414-98932008000100008

Villemor-Amaral, A. E. (2009). Métodos projetivos em avaliações compulsórias: Indicadores e perfis. In C. S. Hutz (Org.), Avanços e polêmicas em avaliação psicológica (pp. 157-174). São Paulo, SP: Casa do Psicólogo.

Wald, B. K., Archer, R. P., \& Winstead, B. A. (1990). Rorschach characteristics of mothers of incest victims. Journal of Personality Assessment, 55(3-4), 417-425.

Weiner, I. B., Exner, J. E., \& Sciara, A. (1996). Is the Rorschach welcome in the courtroom? Journal of Personality Assessment, 67(2), 422-424.

Recebido: $26 / 08 / 2010$

$1^{a}$ revisão: 04/12/2010

Aceite final: 06/07/2011

Silvana Alba Scortegagna é Professora Permanente do Programa de Pós-graduação em Envelhecimento Humano da Universidade de Passo Fundo.

Anna Elisa de Villemor-Amaral é Professora Associada do Programa de Pós-graduação em Psicologia da Universidade São Francisco.

Como citar este artigo:

Scortegagna, S. A., Villemor-Amaral, A. E. (2012). Uso do Rorschach na investigação do abuso sexual infantil. Paidéia (Ribeirão Preto), 22(52), 271-279. doi:10.1590/S0103-863X2012000200013 